\title{
Diacronie
}

Studi di Storia Contemporanea

$N^{\circ} 10,2 \mid 2012$

Digital History: la storia nell'era dell'accesso

\section{Nota introduttiva n. 10 - giugno 2012}

\section{Elisa Grandi e Émilien Ruiz}

\section{(2) OpenEdition}

\section{Journals}

\section{Edizione digitale}

URL: http://journals.openedition.org/diacronie/2762

DOI: 10.4000/diacronie.2762

ISSN: 2038-0925

\section{Editore}

Association culturelle Diacronie

\section{Notizia bibliografica digitale}

Elisa Grandi e Émilien Ruiz, « Nota introduttiva n. 10 - giugno 2012 », Diacronie [Online], N 10, 2 |

2012, Messo online il 29 juin 2012, consultato il 24 septembre 2020. URL : http://

journals.openedition.org/diacronie/2762; DOI : https://doi.org/10.4000/diacronie.2762

Questo documento è stato generato automaticamente il 24 settembre 2020.

Creative Commons License 


\title{
Nota introduttiva n. 10 - giugno 2012
}

\author{
Elisa Grandi e Émilien Ruiz
}

Benché non siano del tutto inedite, negli ultimi anni ${ }^{1}$ si sono moltiplicate le riflessioni incentrate sui mutamenti che si sono prodotti a seguito dell'avvento del digitale nel mestiere di storico e, parallelamente a queste, sono cresciuti numericamente $\mathrm{i}$ contributi relativi alle "Digital humanities" così come all"“umanesimo digitale"3.

2 Con questo decimo numero, Diacronie intende posare un mattone a un edificio ancora in costruzione. Attualmente è correntemente utilizzato il termine

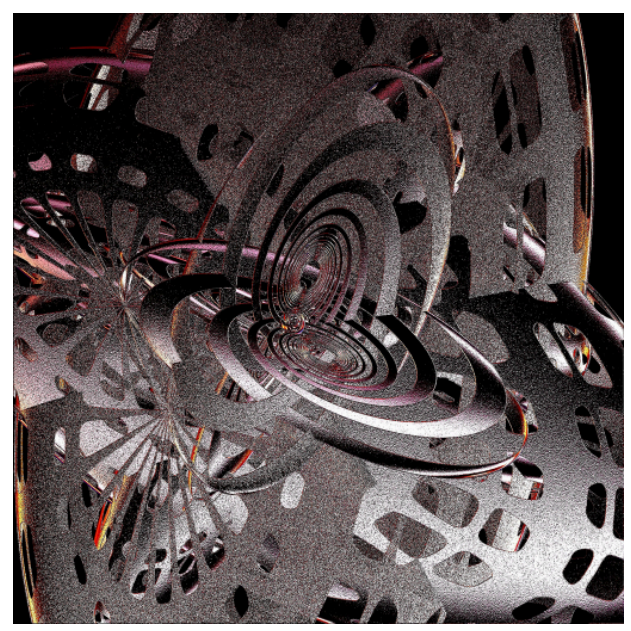
"Digital History" per definire l'utilizzo di strumenti digitali applicati alla ricerca storica, dall'analisi delle fonti alla diffusione dei risultati; con questa etichetta ${ }^{4}$ sono stati creati insegnamenti universitari, sorti principalmente negli Stati Uniti ${ }^{5}$, dove numerosi centri di ricerca si contendono la palma di questa specializzazione ${ }^{6}$.

3 Pertanto l'espressione "Digital History" non è nuova, ma sono ancora da valutare le conseguenze tangibili di un ricorso agli strumenti digitali nel mestiere di storico. Come hanno ricordato recentemente Nicolas Delalande e Julien Vincent, «les discours tenus sur Internet et ses conséquences sur l'élaboration du savoir historique oscillent généralement entre l'enthousiasme, l'inquiétude ou le désintérêt» ${ }^{7}$; quest'ultima posizione viene considerata dai due storici come «la plus fréquente» ${ }^{8}$ e probabilmente non «la moins dommageable» ${ }^{9}$. Con questo numero, Diacronie vuole contribuire a una presa di coscienza da parte degli storici e delle storiche delle trasformazioni prodotte dal digitale sul loro quotidiano lavoro di storici. 
Molti contributi di questo numero prendono in considerazione questo tema da un punto di vista particolare e al contempo universale; tuttavia, in assenza di ricerche di grande respiro, questi saggi non possono che prendere spunto da esperienze personali e concrete. Così i contributi di Brian Sarnacki ${ }^{10}$, Franziska Heimburger e Émilien Ruiz ${ }^{11}$ e l'intervista con Claire Lemercier ${ }^{12}$ da noi realizzata propongono, a partire dalle esperienze di ricerca e di insegnamento dei loro autori, una panoramica delle modificazioni occorse nel mestiere di storico con l'avvento dell'era del digitale: queste riguardano sia le pratiche inerenti l'approccio ai documenti (dalla ricerca al trattamento delle fonti, passando attraverso il lavoro bibliografico), sia le modalità di diffusione della ricerca (dal blog alla rivista scientifica in rete), sia gli scambi scientifici e pedagogici (facilitati enormemente dagli strumenti collaborativi). Tre dimensioni che il dossier permette di illustrare attraverso esempi concreti.

In questo numero di Diacronie, è nostro intento mettere in evidenza la diversità dei possibili approcci alla "Digital History". Molti contributi sono dedicati a strumenti informatici specifici, come i Sistemi di Informazione Geografica (si vedano gli articoli di Cécile Burel, Jordi Rubió e Josep Sitjar ${ }^{13}$ e quello di Nicolas Ferrand ${ }^{14}$ ), le banche-dati, (Lisa Valli ${ }^{15}$ ) e il programma di gestione di dati bibliografici Zotero (Frédéric Clavert ${ }^{16}$ ); ma anche a risorse digitali particolari, utili allo studio della storia economica dell'Italia contemporanea (Matteo Troilo ${ }^{17}$ ), a quello dell'antichità classica (Gianluca Canè ${ }^{18}$ ) o alla storia ambientale (Wilko Graf von Hardenberg e Kimberly Coulter ${ }^{19}$ ).

6 Tutti i contributi testimoniano una duplice necessità. La diversità degli approcci e degli strumenti qui presentati - come in altre pubblicazioni - invitano la comunità degli storici a definire con maggior precisione ciò che è indicato con i termini "Digital History": una semplice, ma profonda, evoluzione del mestiere di storico? Un nuovo campo disciplinare a sé stante? Qualcosa di ancora differente? Per quel che ci riguarda noi sposiamo convintamente l'idea esposta in questo numero da Claire Lemercier, secondo la quale è indispensabile una normalizzazione attraverso la quale l'etichetta di "Digital History" non sia più necessaria. Piuttosto che operare per la costituzione di una sotto-disciplina (come altri hanno tentato di fare, con maggiore o minor successo, con la storia culturale o la sociohistoire) a noi sembra importante partire dal principio che, nell'era digitale, tutta la storia è, almeno in parte, digitale. Questo ci porta alla seconda necessità: quella della formazione di future storiche e storici all'uso di strumenti informatici e di risorse digitali indispensabili a molti, se non a tutti, i ricercatori che si occupano di storia. Oggi quella di offrire agli storici in fieri i mezzi per orientarsi nel nuovo mondo digitale è una sfida che non è stata ancora raccolta dalle istituzioni culturali e universitarie. Questo numero di Diacronie da solo non saprà rispondere esaustivamente a questioni di così vasta portata, ma noi ci auguriamo che possa contribuire a suscitare riflessioni sulle trasformazioni in corso nel nostro mestiere. 


\section{NOTE}

1. Si vedano, tra le publicazioni recenti: GENET, Jean-Philippe, ZORZI, Andrea (a cura di), Les historiens et l'informatique: un métier à réinventer, Rome, École française de Rome, 2011; GALLINI, Stefania, NOIRET, Serge (a cura di), «La Historia digital en la era del Web 2.0. Introducción al dossier Historia Digital», in Historia Critica, 43, 2011, URL: < http://historiacritica.uniandes.edu.co/ indexar.php?c=Revista+No+43 > [consultato il 26 giugno 2012]; DELALANDE, Nicolas, VINCENT, Julien (a cura di), Revue d'histoire moderne et contemporaine: Le métier d'historien à l'ère numérique: nouveaux outils, nouvelle épistémologie?, 58, 4bis/2011; CLAVERT, Frédéric, NOIRET, Serge (a cura di), L'histoire contemporaine à l'ère digitale, Bruxelles, PIE-Peter Lang, 2012 [Opera in corso di pubblicazione, frutto dell'omonimo colloquio tenuto nell'ottobre 2009 all'université du Luxembourg].

2. DACOS, Marin, «Manifeste des Digital humanities», in ThatCamp Paris 2010, URL: < http:// tcp.hypotheses.org/443 > [consultato 26 giugno 2012].

3. DOUEIHI, Milad, Pour un humanisme numérique, Paris, Le Seuil, 2011; NUMERICO, Teresa, FIORMONTE, Domenico, TOMASI, Francesca, L'umanista digitale, Bologna, Il Mulino, 2010.

4. Si veda, a titolo d'esempio, l'annuncio largamente diffuso dall'Université du Luxembourg: «The University of Luxembourg is recruiting an Associate Professor or Professor in Digital History», in Calenda, URL: < http://calenda.revues.org/nouvelle24595.html > [consultato il 26 giugno 2012].

5. L'espressione sembra sia nata alla fine degli anni Novanta con la fondazione del Virginia Center for Digital History nel 1998, sotto la guida di Edward L. Ayers (rettore dell'Università di Richmond dal 2007). Il primo riferimento in una pubblicazione ufficiale è apparentemente datato al 1999: AYERS, Edward L., «The Pasts and Futures of Digital History», Virginia Center for Digital History, URL: < http://www.vcdh.virginia.edu/PastsFutures.html > [consultato il 26 Giugno 2012]. Si veda anche COHEN, Daniel, ROSENZWEIG, Roy, Digital History: A Guide to Gathering, Preserving, and Presenting the Past on the Web, Ann Arbor, University of Pennsylvania Press, 2005.

6. Come il Roy Rosenzweig Center for History and New Media dell'Università Georges Mason < http://chnm.gmu.edu/ > o il Virginia Center for Digital History précité, de l'Université de Virginie < http://www.vcdh.virginia.edu/ > Per altri esempi si vedano le sezioni «Digital history center» e «Digital history projects» in «Digital history», Wikipedia, the free encyclopedia, URL: < http://en.wikipedia.org/ > [consultati il 26 Giugno 2012].

7. DELALANDE, Nicolas, VINCENT, Julien, «Portrait de l'historien-ne en cyborg», in Revue d'histoire moderne et contemporaine, 58, 4bis/2011, pp. 5-29.

8. Ibidem.

9. Ibidem.

10. SARNACKI, Brian, «Digitizing the Seminar Paper. A graduate student perspective on how digital tools shape scholarship", Diacronie. Studi di Storia Contemporanea : Digital History: la storia nell'era dell'accesso, N. 10, 2|2012, URL: < http://www.studistorici.com/2012/06/29/ sarnacki_numero_10/ >.

11. HEIMBURGER, Franziska, RUIZ, Émilien, «Has the Historian's craft gone digital? Some observations from France», Diacronie. Studi di Storia Contemporanea : Digital History: la storia nell'era dell'accesso, N. 10, 2|2012, URL: < http://www.studistorici.com/2012/06/29/heimburgerruiz_numero_10/ >.

12. GRANDI, Elisa, RUIZ, Émilien, «Ce que le numérique fait à l'historien.ne. Entretien avec Claire Lemercier», Diacronie. Studi di Storia Contemporanea : Digital History: la storia nell'era dell'accesso, N. 10, 2|2012, URL: < http://www.studistorici.com/2012/06/29/grandi_numero_10/ >.

13. BUREL, Cécile, RUBIÓ, Jordi, SITJAR, Josep, «Los SIG como instrumento para el análisis de las migraciones: el ejemplo del éxodo catalán de 1936», Diacronie. Studi di Storia Contemporanea : Digital 
History: la storia nell'era dell'accesso, N. 10, 2|2012, URL: < http://www.studistorici.com/2012/06/29/ burel-rubio-sitjar_numero_10/ >.

14. FERRAND, Nicolas, «Développement et mise en œuvre d'un Système d'Information Géographique pour l'étude de l'expansion pavillonnaire dans l'agglomération lyonnaise depuis 1950», Diacronie. Studi di Storia Contemporanea : Digital History: la storia nell'era dell'accesso, N. 10, 2 | 2012, URL: < http://www.studistorici.com/2012/06/29/ferrand_numero_10/ >.

15. VALLI, Lisa, «Vecchie abitudini e nuovi strumenti. Un sistema informativo per lo studio delle vicende architettoniche del Palazzo Ducale di Mantova», Diacronie. Studi di Storia Contemporanea : Digital History: la storia nell'era dell'accesso, N. 10, 2|2012, URL: < http://www.studistorici.com/ 2012/06/29/valli_numero_10/ >.

16. CLAVERT, Frédéric, «Au-delà de la gestion des références bibliographiques : Zotero», Diacronie. Studi di Storia Contemporanea : Digital History: la storia nell'era dell'accesso, N. 10, 2|2012, URL: < http://www.studistorici.com/2012/06/29/clavert_numero_10/ >.

17. TROILO, Matteo, «Il digitale che diventa archivio. Le fonti on-line per la storia economica italiana», Diacronie. Studi di Storia Contemporanea : Digital History: la storia nell'era dell'accesso, N. 10, 2|2012, URL: < http://www.studistorici.com/2012/06/29/troilo_numero_10/ >.

18. CANÈ, Gianluca, «La lezione di Prometeo e il filologo nell'era digitale. Rassegna di risorse informatiche per lo studio dell'antichità classica», Diacronie. Studi di Storia Contemporanea : Digital History: la storia nell'era dell'accesso, N. 10, 2|2012, URL: < http://www.studistorici.com/2012/06/29/ cane_numero_10/ >.

19. GRAF VON HARDENBERG, Wilko, COULTER, Kimberly, «Navigare la storia: considerazioni sulla creazione di un portale di storia ambientale», Diacronie. Studi di Storia Contemporanea : Digital History: la storia nell'era dell'accesso, N. 10, 2|2012, URL: < http://www.studistorici.com/2012/06/29/ grafvonhardenberg-coulter_numero_10/ >.

\section{AUTORI}

\section{ELISA GRANDI}

Elisa Grandi è Dottoranda in Storia economica e storia contemporanea dell'America Latina all'Université Paris VII - Denis Diderot in cotutela con l'Università di Bologna; è inoltre membro del laboratorio SEDET. Insegna metodologia della ricerca storica, critica delle fonti e storia dei Paesi non occidentali à Paris Diderot. Tra i suoi ambiti di ricerca vi sono la storia transnazionale, l'economia dello sviluppo, la social network analysis e la digital history. Si occupa di diversi progetti legati alle Digital Humanities in collaborazione con l'Ecole des Hautes Etudes di Parigi. Sta conducendo una ricerca dal titolo: Reti Sociali e cambiamenti istituzionali. International economic advising, amministrazione pubblica e riforme economiche in Colombia (1949-1970) sotto la direzione di Zacarias Moutoukias. Nell'ambito delle sue ricerche ha rivolto la sua attenzione alla metodologia della ricerca storica, in particolare all'applicazione di tecniche proprie della ricerca economica e della sociologia alle scienze storiche. Dopo essere stata Visiting Student alla Duke University e Visiting Scholar alla New School of Social Research, è attualmente borsista del governo francese Eiffel-Excellence presso l'Université Paris 7 - Denis Diderot. Dal 2002 al 2007 è stata allieva del Collegio Superiore dell'Università di Bologna. Nel 2009 ha svolto il ruolo di consulente editoriale 
e traduttrice per conto delle Mayol Ediciones.

URL: < http://www.studistorici.com/2010/12/07/elisa_grandi/ >

\section{ÉMILIEN RUIZ}

Émilien Ruiz est doctorant en histoire contemporaine à l'École des Hautes Études en Sciences Sociales (EHESS). Sa thèse Trop de fonctionnaires? La question des effectifs de l'État dans la France du XXe siècle porte sur divers aspects de l'évolution du nombre des fonctionnaires de l'État français des années 1880 aux années 1980. Il a enseigné la méthodologie de la recherche en histoire, l'histoire contemporaine et l'informatique pour les sciences historiques à l'EHESS et à l'Université Paris Diderot. Depuis janvier 2012, il est assistant de Paul-André Rosental à Sciences po. Il anime deux blogs scientifiques: La Boite à Outils des Historiens (avec Franziska Heimburger), sur les outils informatique pour l'histoire; Devenir historien-ne, sur la méthodologie de la recherche en histoire. Parmi ses publications: «Compter: l'invention de la statistique des fonctionnaires en France (années 1890-1930)», in BEZES, Philippe, JOIN-LAMBERT, Odile (dir.), «Comment se font les administrations», Sociologie du Travail, 52, 2/2010, pp. 212-233. URL: < http://www.studistorici.com/2010/12/07/emilien_ruiz/ > 